\section{Attention to TEN}

Dear Editor,

The authors of the article "Toxic Epidermal Necrolysis Managed with Immunoglobulin" MJAFI 2008; 64: 272-3 deserves kudos for their success. But my heart felt compliments are reserved for the patient who survived not only the potentially fatal disease but also the frightening therapeutic exercise.

The role of antibiotics in treating a patient of TEN is circumspect when 'multiple blood, urine and fluid from blister cultures were sterile' as reported. Antibiotic therapy is recommended only when signs of sepsis occur; and if commenced prophylactically or empirically, can lead to emergence of resistant strains and increased mortality [1].

The choice of antibiotic also deserves attention. The authors have surmised that cefixime, a third generation cephalosporin and a beta-lactam drug, may have caused TEN. Yet, piperacillin - another beta-lactam drug was exhibited, which has issues of crosssensitisation with cephalosporin. Though some workers report less cross-reactivity between penicillins and the newer generation cephalosporins [2], it is well known that cross-sensitisation can cause TEN within different classes of beta-lactams like penicillins and cephalosporins [3]. Recently, allergic reaction to piperacillin has been reported in a case of ceftrioxone (another third generation cephalosporin) - induced TEN, suggesting an undelineated mechanism of cross sensitivity and hypersensitivity between âlactams [4].

With the clinical and biochemical data available, the basis of arriving at SCORTEN value of three is not clear. Histopathology, if performed, could have corroborated by demonstration of necrotic epidermal cells and quantification of dermal mononuclear cell infiltrate, the density of which correlates with the severity of TEN and is almost as accurate as SCORTEN in predicting outcome [5].

It was also too enthusiastic to propound IVIg as a panacea for

Toxic Epidermal Necrolysis Managed with Immunoglobulin

Reply

We thank the reader for critical appraisal of our report and kind remarks. The reply to queries raised by him is as follows;

Our patient had presented with fever and was receiving antibiotics at the time of presentation which was the reason of negative cultures. Sepsis is the most debilitating complication in TEN and is one of the leading causes of mortality [1]. Patient had onset of TEN following therapy for fever, cough and sore throat and was continuing antibiotics till consultation in the emergency at our centre. In presence of $>80 \%$ body surface area involvement with purulent mucositis of the oral and urethra and urinalysis full of pus cells, infection was very much evident which prompted us to continue with changed antibiotics despite culture reports being negative. Lastly, offering antibiotic therapy after obtaining culture positive reports and or signs of septic shock seems purely academic and sometimes dangerous in day to day practice.
TEN. While literature abounds with successes with IVIg, there are many well planned studies which do not echo the same view[6].

The most crucial management in a case of TEN is discontinuation of the offending drug and intensive supportive care [6]. Sadly, both of these seem to have somehow missed the focus here.

\section{References}

1. Schultz JT, Sheridian RL, Ryan CM, et al. A 10-year experience with toxic epidermal necrolysis. J Burn Care Rehabil 2000; 21: 199-204.

2. Banks JR, Arnold. Immediate Allergic Reactions to Cephalosporins: Cross-Reactivity and Selective Responses. Pediatrics 2002; 110: 439-40.

3. Paquet P, Jacob E, Damas P, Pierard GE. Recurrent fatal druginduced toxic epidermal necrolysis (Lyell's syndrome) after putative beta lactam cross reactivity: case report and scrutiny of antibiotic imputability. Crit Care Med 2002; 30: 2580-3.

4. Lam A, Randhawa IP, Klaustermeyer W. Cephalosporin Induced Toxic Epidermal Necrolysis and Subsequent Penicillin Drug Exanthem. Allergology International 2008; 57: 281-4.

5. Quinn AM, Brown K, Bonish BK, Curry J, Gordon KB, Sinacore J, et al. Uncovering histological criteria with prognostic sign in toxic epidermal necrolysis. Arch Dermatol 2005; 141 : 683-7.

6. Pereira FA, Mudgil AV, Rosmarin DM. Toxic Epidermal Necrolysis. J Am Acad Dermatol 2007; 56: 181-200.

Contributed by

Wg Cdr Sanjiv Grover

Associate Professor (Derm \& Ven), Department of Dermatology, Command Hospital (SC), Pune - 411040.

IgE mediated hypersensitivities are well known to be cross reactive between cephalosporins and penicillins, however, non IgE mediated hypersensitivities usually are not cross reactive. Patients with a prior immediate hypersensitivity reaction to penicillin should generally not be treated with cephalosporin unless: Penicillin or cephalosporin skin testing, if available, is negative or a graded challenge with the cephalosporin can be given under careful observation by an allergist. A history of a rash or unclear reaction to penicillin does not necessarily preclude administration of cephalosporins [2]. Moreover, there is not a single report of TEN attributable to piperacillin till date on medline search. Only single case report putatively linking cephalosporin hypersensitivity to Meropenem induced TEN has been reported [3]. This does not preclude the use of other beta lactam antibiotics, it just merits caution. Compared to sulfonamides antibiotics, other sulfonamides 